\title{
Management and Treatment of Hepatocellular Carcinoma with Immunotherapy: A Review of Current and Future Options
}

\author{
Shima Ghavimi, Tehila Apfel, Hamed Azimi, Alana Persaud and Nikolaos T. Pyrsopoulos* \\ Division of Gastroenterology and Hepatology, Department of Medicine, Rutgers New Jersey Medical School, Newark, NJ, USA
}

\begin{abstract}
With mortality rates of liver cancer doubling in the last 20 years, this disease is on the rise and has become the fifth most common cancer in men and the seventh most common cancer in women. Hepatocellular carcinoma (HCC) represents approximately $90 \%$ of all primary liver cancers and is a major global health concern. Patients with HCC can be managed curatively with surgical resection or with liver transplantation, if they are diagnosed at an early stage. Unfortunately, most patients with HCC present with advanced stages of the disease and have underlying liver dysfunction, which allows only $15 \%$ of patients to be eligible for curative treatment. Several different treatment modalities are available, including locoregional therapy radiofrequency ablation, microwave ablation, percutaneous ethanol injection, trans-arterial chemoembolization, transarterial radio-embolization, cryoablation, radiation therapy, stereotactic radiotherapy, systemic chemotherapy, molecularly targeted therapies, and immunotherapy. Immunotherapy has recently become a promising method for inhibiting HCC tumor progression, recurrence, and metastasis. The term "Immunotherapy" is a catch-all, encompassing a wide range of applications and targets, including HCC vaccines, adoptive cell therapy, immune checkpoint inhibitors, and use of oncolytic viruses to treat HCC. Immunotherapy in HCC is a relatively safe option for treating patients with advanced disease in the USA who are either unable to receive or failed sorafenib/lenvatinib therapy and thus may offer an additional survival benefit for these patients. The purpose of this review is to elaborate on some of the most recent advancements in immunotherapy.
\end{abstract}

Citation of this article: Ghavimi S, Apfel T, Azimi H, Persaud A, Pyrsopoulos NT. Management and treatment of hepatocellular carcinoma with immunotherapy: A review of current and future options. J Clin Transl Hepatol 2020;8 (2):168-176. doi: 10.14218/JCTH.2020.00001.

Keywords: Carcinoma; Hepatocellular; Immunotherapy; Immunotherapy; Adoptive; Nivolumab.

Abbreviations: $A C T$, adoptive cell therapy; CAR, chimeric antigen receptor CTLA-4, cytotoxic T Iymphocyte-associated protein 4; HCC, hepatocellular carcinoma; MELD, model for end-stage liver disease; PD-1, programmed cell death-1; $\mathrm{PD}-\mathrm{L} 1$, programmed death-ligand 1; TACE, trans-arterial chemoembolization; VEGF, vascular endothelial growth factor.

Received: 6 January 2020; Revised: 13 May 2020; Accepted: 16 May 2020

* Correspondence to: Nikolaos T. Pyrsopoulos, Division of Gastroenterology and Hepatology, Department of Medicine, Rutgers New Jersey Medical School, 185 South Orange Avenue, H-538, Newark, NJ 07103, USA. Tel: +1-973-972-5252, Fax: +1-973-9723144, E-mail: pyrsopni@njms.rutgers.edu

\section{Introduction}

With mortality rates of liver cancer doubling in the last 20 years, this disease is on the rise and has become the fifth most common cancer in men and the seventh most common cancer in women. ${ }^{1}$ Currently, hepatocellular carcinoma (HCC) represents approximately $90 \%$ of primary liver cancers and is a major global health concern. ${ }^{2}$ There are many risk factors responsible for the development of $\mathrm{HCC}$, such as viral infections, hereditary hemochromatosis, non-alcoholic fatty liver disease, increased alcohol-related liver disease, and cirrhosis, among others. ${ }^{3}$ HCC development is a complex process involving multiple factors and pathways that lead to changes in gene expression, immune interactions, and changes in the tumor microenvironment that ultimately cause hepatocarcinogenesis. ${ }^{4}$

Screening for HCC in high-risk populations has become the standard of care, requiring imaging with ultrasound, computerized tomography, or magnetic resonance imaging every 612 months. ${ }^{5}$ Laboratory tests, including mainly those for alpha feto-protein, the lectin Lens culinaris agglutinin-bound fraction of alpha feto-protein-3, and des-gamma-carboxy prothrombin, are used in conjunction with imaging to establish the diagnosis. ${ }^{6,7}$ Multiphasic imaging modalities, such as computerized tomography and magnetic resonance imaging, are used for detection and diagnosis of HCC. Liver biopsy is not necessary once the Liver Imaging Reporting and Data System (known as 'Li-Rads') has been deemed to be category 5 , which is diagnostic for HCC. ${ }^{8}$

If a lesion is deemed to be HCC, the available options for management are varied and depend on multiple factors, including the number of lesions, their size, the presence of extrahepatic spread, and the severity of the patient's underlying liver disease. ${ }^{9}$ The recommendation from The European Association for the Study of Liver panel of experts is to consider the following four related aspects to determine treatment options: tumor stage, degree of liver function impairment, general condition of the patient, and treatment efficacy. ${ }^{10,11}$ Fig. 1 depicts the Barcelona Clinic Liver Cancer system, which is one of the most commonly used algorithms to assist in determining treatment options based on the aforementioned factors. ${ }^{11-14}$ To summarize, patients diagnosed as stage 0 , with very early $\mathrm{HCC}$, are ideal candidates for ablation or resection. ${ }^{11}$ Patients who are deemed to be stage $A$, with early HCC, are candidates for radical therapies, including hepatoma resection, liver transplantation, or interventional radiology procedures. ${ }^{10,11}$ Patients at stage $B$, with intermediate HCC, may benefit from chemoembolization. ${ }^{10,11}$ Patients at stage $\mathrm{C}$, who already have advanced HCC, are only candidates for systemic therapy if their performance status is acceptable; otherwise, they are managed with the best 
supportive care available. ${ }^{11}$ Approximately $85 \%$ of patients with HCC are diagnosed at later stages or have underlying liver dysfunction, limiting their treatment options. ${ }^{9}$ These patients usually have a very poor prognosis, with survival of less than 1 year. ${ }^{15}$ In patients treated with resection or ablation, tumor recurrence (both true recurrence due to dissemination and de novo tumors) is unfortunately common and is seen in up to $70 \%$ of patients 3-5 years after treatment. ${ }^{16}$

Liver transplantation is an important treatment modality for patients who meet Milan criteria (a single HCC nodule of 2$5 \mathrm{~cm}$ or $3 \mathrm{HCC}$ nodules each $\leq 3 \mathrm{~cm}$ in diameter) or who undergo down-staging of their tumors to be within the Milan criteria. ${ }^{17-20}$ Studies have shown that patients who met Milan criteria and received a liver transplant had survival rates exceeding $70 \%$ at 5 years, with recurrence in less than $15 \%$ of patients. ${ }^{21}$ Approximately $30-40 \%$ of patients on the liver transplantation waitlist are patients who have received model for end-stage liver disease (MELD) exception points for HCC. ${ }^{22}$ They receive these points 6 months after listing and then receive an incremental increase in their MELD points every 3 months until the maximum MELD exception point allowance is reached (that being 34). ${ }^{23,24}$ MELD exception points give patients an increased chance of receiving a liver but they do not guarantee a liver to all listed patients. Therefore, additional treatments for HCC are greatly needed.

In 1891, the surgeon William Coley injected streptococcal organisms into a patient with inoperable osteosarcoma, successfully stimulating the immune system and leading to tumor regression and thus fathering the field of immunotherapy. ${ }^{25}$ Since then, there have been many achievements in use of immunotherapy to fight cancer and in the development of a broad range of therapeutic applications, including the use of gene therapy, oncolytic viruses, cytokines, adoptive cell therapy, vaccines, and immune checkpoint inhibitors to fight cancer. $^{25}$

Immunotherapy has recently become a new promising method for inhibiting HCC tumor progression, recurrence, and metastasis. ${ }^{26,27}$ "Immunotherapy" is a catch-all term, encompassing a wide range of applications and targets, including HCC vaccines, adoptive cell therapy (ACT), immune checkpoint inhibitors, and use of oncolytic viruses to treat HCC. These approaches have often shown initial success in treating other types of cancers, with potential to be similarly successful in treating HCC. In this review, we will discuss some of the most recent advancements in immunotherapy for HCC.

\section{Tumor immunology}

Research has shown that cancer cells are able to escape from immunological surveillance and suppress the activation of immunocompetent cells (immune suppression), thereby allowing for their continued growth. ${ }^{27}$ Cancer immunoediting is a proposed mechanism to explain how tumors evade the

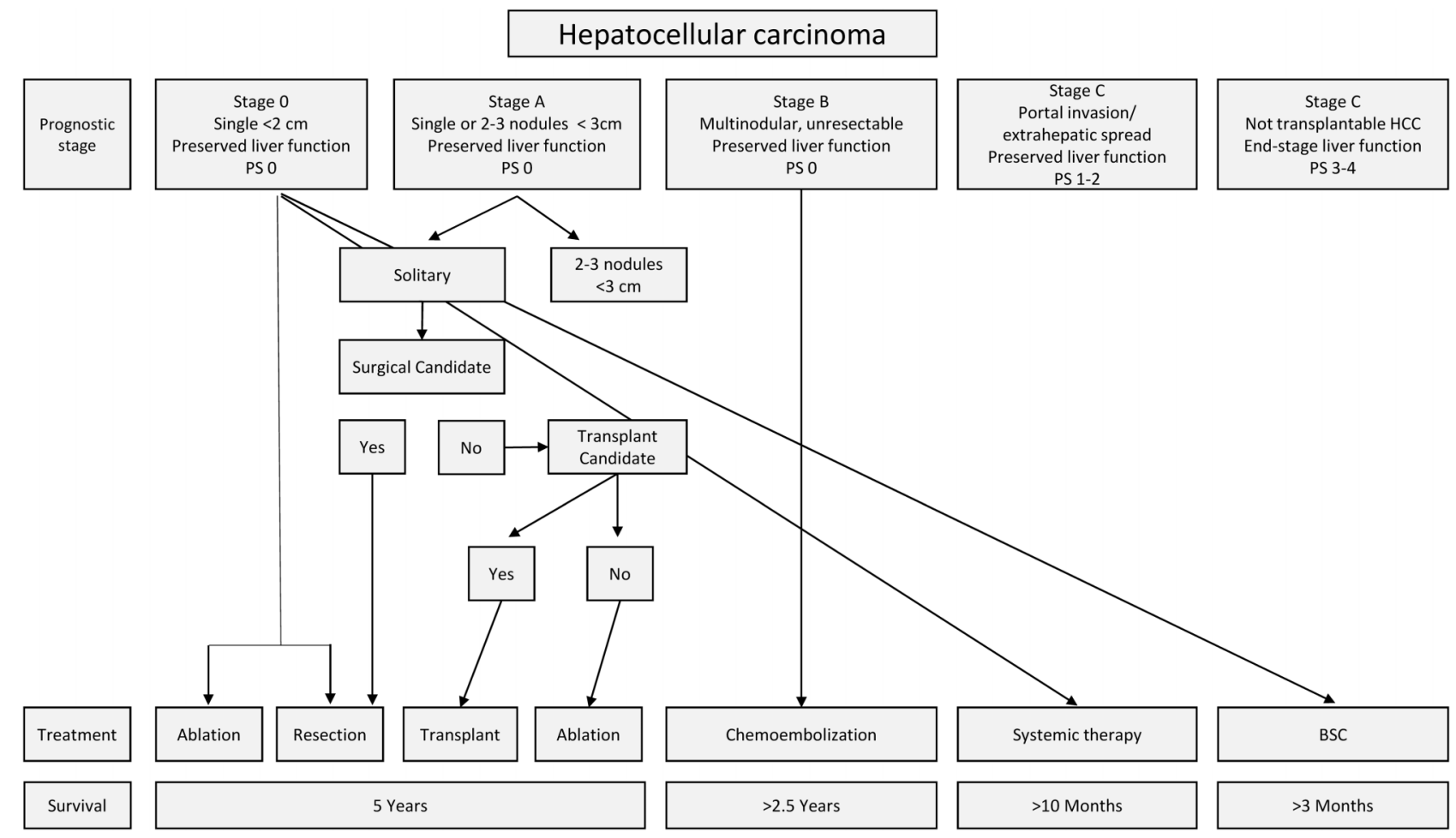

Fig. 1. Hepatocellular carcinoma treatment in patients diagnosed with hepatocellular carcinoma.

Modified Barcelona Clinic Liver Cancer (BCLC) staging and treatment strategy: The BCLC system recommends pathways for treatment based on prognostic stages. The stage is determined by the number of lesions and their size, evidence of extrahepatic spread/portal invasion, performance status (ps), preserved liver function, and evidence of decompensated liver disease (usually determined by the Child-Pugh classification or the model for end-stage liver disease score). As noted, there are multiple treatment options, including resection, transplantation, chemoembolization, ablation, systemic therapy or best support care, which is essentially palliative care. Survival is predicted based on what initial therapy was chosen. ${ }^{11}$ 
immune system, consisting of three sequential phases: elimination, equilibrium, and escape. ${ }^{28}$ In the elimination phase, innate and adaptive immunity work together to destroy developing HCC long before it becomes clinically apparent. If this phase is not successful, the cancer cell variant may then enter the equilibrium phase, in which its growth is prevented by immunologic mechanisms. ${ }^{28}$ Important players from the adaptive immune system, T cells, interleukin-12, and interferon- $\alpha$ suppress the growth of cancer cells during this phase. ${ }^{28}$ The equilibrium state may represent an end stage of the cancer immunoediting process, creating occult cancers that do not grow any larger and are clinically insignificant during the lifetime of the host. ${ }^{28}$ However, because of this constant immune selection pressure placed on "genetically unstable" tumor cells, new cell variants can become (1) unrecognizable to the adaptive immune system, due to antigen loss and defects in antigen processing or presentation, (2) insensitive to immune effector mechanisms, or (3) able to induce an immunosuppressive state within the tumor microenvironment. ${ }^{28}$ These tumor cells may then enter the escape phase, in which their growth is no longer controlled by the immune system and clinically significant disease develops. 28

An important escape mechanism allows tumor cells to upregulate their own expression of immune checkpoint molecules, including the programmed cell death-1 (PD-1) protein that binds to the programmed death-ligand 1 (PD-L1) and stimulates peripheral $\mathrm{T}$ cell depletion. ${ }^{29}$ Another important checkpoint molecule, cytotoxic $T$ lymphocyte-associated protein 4 (CTLA-4), is found on the surface of T cells and can be activated by tumor cells, leading to down-regulation of T cells. ${ }^{27}$ Interestingly, initial studies done by Duffy et al. ${ }^{30}$ in 2017 showed that in liver biopsies of patients treated with tremeliomumab, an anti-CTLA-4 antibody, there was an increase in cytotoxic T cells, demonstrating that treatment with such molecules increased activity of the immune system. Monoclonal antibodies to PD-1, PD-L1, and CTLA-4 are called immune checkpoint inhibitors and have become an important part of immunotherapy treatments for many cancers, including melanoma, non-small cell lung cancer, and colorectal cancer, and are now emerging as valuable treatments in HCC. 29,31

\section{Checkpoint inhibitors}

Checkpoint inhibitors are currently the most successful immunotherapy treatment for $\mathrm{HCC}^{27}$ As noted in Tables 1 and 2, and illustrated in Fig. 2, there are multiple novel treatments available and many active clinical trials investigating further checkpoint inhibitors.

\section{Nivolumab}

Promising results were reported in 2017 from the Checkmate 040 phase I/II trial which looked into survival rates for nivolumab, an anti-PD-1 antibody, used in the treatment of advanced HCC. ${ }^{32}$ A dose-escalation and expansion phase was implemented in patients who met strict inclusion criteria, with clinically less severe underlying liver dysfunction. ${ }^{32}$ Treatment in 262 patients yielded an acceptable safety profile and promising efficacy, and based on these results, the Food and Drug Administration fast-tracked the approval of nivolumab for the treatment of patients diagnosed with HCC who had been previously treated with sorafenib. ${ }^{27}$ Another small phase II trial investigating nivolumab alone versus nivolumab and ipilimumab is currently underway, with preliminary results demonstrating a good safety profile and that treatment does cause delay to surgical resection. ${ }^{33}$

Table 1. Results of selected studies testing immune checkpoint inhibitors in hepatocellular carcinoma. Adapted from Pinter and Peck-Radosavljevic. ${ }^{25}$

\begin{tabular}{|c|c|c|c|c|c|c|c|}
\hline $\begin{array}{l}\text { Trial and } \\
\text { year }\end{array}$ & $\begin{array}{l}\text { Treatment (number } \\
\text { of patients) }\end{array}$ & Target IT & $\begin{array}{l}\text { Prior sorafenib } \\
\text { treatment, } \%\end{array}$ & $\begin{array}{l}\text { ORR/ } \\
\text { DCR, \% }\end{array}$ & $\begin{array}{l}\text { TTP/PFS } \\
\text { in months }\end{array}$ & $\begin{array}{l}\text { OS in } \\
\text { months }\end{array}$ & Reference \\
\hline $\begin{array}{l}\text { Sangro } \\
2013\end{array}$ & Tremelimumab (21) & Anti-CTLA-4 & 23.8 & $17.6 / 76.4$ & $6.48 / N R$ & 8.2 & 49 \\
\hline Duffy 2017 & $\begin{array}{l}\text { Tremelimumab }+ \\
\text { subtotal ablation (32) }\end{array}$ & Anti-CTLA-4 & 65.6 & $26.3 / \mathrm{NR}$ & 7.4/NR & 12.3 & 30 \\
\hline $\begin{array}{l}\text { El-Khoueiry } \\
2017\end{array}$ & Nivolumab (80) & Anti-PD-1 & 0 & $22.5 / 62.5$ & NR/NR & 28.6 & 32 \\
\hline $\begin{array}{l}\text { El-Khoueiry } \\
2017\end{array}$ & Nivolumab (182) & Anti-PD-1 & 100 & $18.7 / 62.6$ & NR/NR & 15.6 & 32 \\
\hline $\begin{array}{l}\text { Wainberg } \\
2017\end{array}$ & Durvalumab (40) & Anti-PD-L1 & 92.5 & $10 / 32.5$ & $\mathrm{NR} / 2.7$ & 13.2 & 73 \\
\hline Kelley 2017 & $\begin{array}{l}\text { Durvalumab + } \\
\text { tremelimumab (40) }\end{array}$ & $\begin{array}{l}\text { Anti-PD-L1 + } \\
\text { Anti-CTLA-4 }\end{array}$ & 75 & $25 / 57.5$ & NR/NR & NR & 74 \\
\hline Zhu 2018 & $\begin{array}{l}\text { Pembrolizumab } \\
\text { (104) }\end{array}$ & Anti-PD-1 & 100 & $17.3 / 61.5$ & $N R / 4.9$ & 12.9 & 37 \\
\hline Ikeda 2018 & $\begin{array}{l}\text { Pembrolizumab + } \\
\text { lenvatinib (30) }\end{array}$ & Anti-PD-1 & 13.3 & $42.3 / 100$ & NR/9.7 & NR & 42 \\
\hline Stein 2018 & $\begin{array}{l}\text { Atezolizumab }+ \\
\text { bevacizumab (43) }\end{array}$ & $\begin{array}{l}\text { Anti-PD-1 + } \\
\text { anti-VEGF }\end{array}$ & 0 & $65 / 96 \%$ & $N R / N R$ & NR & 51 \\
\hline
\end{tabular}

Abbreviations: CTLA-4, cytotoxic T lymphocyte-associated protein 4; DCR, disease control rate; IT, immunotherapy; NR, not reported; ORR, overall response rate; OS overall survival; PD-1, programmed cell death-1; PD-L1, programmed cell death 1-ligand 1; PFS, progression-free survival; TTP, time to progression. 
Ghavimi S. et al: Management of HCC with immunotherapy

Table 2. Ongoing phase III trials testing immune checkpoint inhibitors in hepatocellular carcinoma

\begin{tabular}{|c|c|c|c|c|c|}
\hline Drug & Target of IT & Setting & $\begin{array}{l}\text { ClinicalTrials } \\
\text { Identifier }\end{array}$ & Status & $\begin{array}{l}\text { Primary } \\
\text { completion }\end{array}$ \\
\hline Nivolumab vs. placebo & Anti-PD-1 & Curative adjuvant & $\underline{\text { NCT03383458 }}$ & Recruiting & Q1 2022 \\
\hline Nivolumab + TACE & Anti-PD-1 & Curative adjuvant & NCT03143270 & Recruiting & Q1 2019 \\
\hline Nivolumab + TACE & Anti-PD-1 & Curative, adjuvant & NCT03572582 & Recruiting & Q3 2022 \\
\hline Nivolumab vs. sorafenib & Anti-PD-1 & Palliative, 1st-line & NCT02576509 & Recruiting & Q3 2017 \\
\hline $\begin{array}{l}\text { Durvalumab } \pm \text { tremelimumab } \\
\text { vs. sorafenib }\end{array}$ & $\begin{array}{l}\text { Anti-PD-L1 + } \\
\text { Anti-CTLA-4 }\end{array}$ & Palliative, 1st-line & NCT03298451 & Recruiting & Q1 2020 \\
\hline $\begin{array}{l}\text { Atezolizumab + bevacizumab } \\
\text { vs. sorafenib }\end{array}$ & Anti-PD-L1 & Palliative, 1 st-line & NCT03434379 & Recruiting & Q2 2021 \\
\hline Pembrolizumab vs. placebo & Anti-PD-1 & Palliative, 2nd-line & NCT02702401 & $\begin{array}{l}\text { Active, not } \\
\text { recruiting }\end{array}$ & Q1 2019 \\
\hline Pembrolizumab + TACE & Anti-PD-1 & Curative, 2nd-line & NCT03397654 & Recruiting & Q4 2019 \\
\hline Pembrolizumab vs. placebo & Anti-PD-1 & Palliative, 2nd-line & NCT03062358 & Recruiting & Q4 2019 \\
\hline $\begin{array}{l}\text { Pembrolizumab vs. best } \\
\text { supportive care }\end{array}$ & Anti-PD-1 & Palliative, 2nd-line & NCT02702401 & $\begin{array}{l}\text { Active, not } \\
\text { recruiting }\end{array}$ & Q4 2020 \\
\hline Tislelizumab vs. sorafenib & Anti-PD-1 & Curative, 1st-line & NCT03412773 & Recruiting & Q4 2022 \\
\hline Atezolizumab + bevacizumab & $\begin{array}{l}\text { Anti-PD-L1 + } \\
\text { anti-VEGF }\end{array}$ & Curative, 1st-line & NCT03434379 & Recruiting & Q2 2022 \\
\hline Ipilimumab + nivolumab & $\begin{array}{l}\text { Anti-CTLA-4 + } \\
\text { Anti-PD-1 }\end{array}$ & Curative, adjuvant & NCT03682276 & Recruiting & Q4 2022 \\
\hline Nivolumab + yttrium-90 & Anti-PD-1 & Curative, adjuvant & NCT03033446 & Recruiting & Q4 2019 \\
\hline $\begin{array}{l}\text { Pembrolizumab + talimogene } \\
\text { laherparepvec }\end{array}$ & $\begin{array}{l}\text { Anti-PD-1 }+ \\
\text { Oncolytic Viral }\end{array}$ & Curative, adjuvant & NCT02509507 & Recruiting & Q3 2021 \\
\hline $\begin{array}{l}\text { durvalumab }+ \text { tremelimumab } \\
+ \text { radiotherapy }\end{array}$ & $\begin{array}{l}\text { Anti-PD-L1 }+ \\
\text { Anti-CTLA-4 }\end{array}$ & Curative, adjuvant & NCT03482102 & Recruiting & Q4 2025 \\
\hline
\end{tabular}

Abbreviations: CTLA-4, cytotoxic T lymphocyte-associated protein 4; IT, immunotherapy; PD-1, programmed cell death-1; PD-L1, programmed cell death 1-ligand 1.

Checkpoint 459 is a phase III study comparing nivolumab and sorafenib as first-line treatment for HCC. ${ }^{34}$ Preliminary data was released in June 2019, which showed that the overall survival failed to meet statistical significance (hazard ratio of $0.85 ; 95 \%$ confidence interval, $0.72-1.02 ; p=0.0752$ ). The data reportedly did show a trend towards an overall survival improvement with nivolumab versus sorafenib. ${ }^{34}$ At the time of this publication, Bristol-Myers Squibb has not yet released the full data. ${ }^{34}$ There are also currently other ongoing trials investigating nivolumab as a single agent in CheckMate-9DX and also in combination with ipilimumab for previously-treated patients with HCC. $26,35,36$

\section{Pembrolizumab}

While nivolumab was investigated as a first-line treatment option for HCC, another anti-PD-1 antibody, pembrolizumab, is being developed as a second-line treatment after initial treatment with tyrosine kinase inhibitors have failed or were not tolerable. ${ }^{27}$ In a phase II trial, patients with advanced liver cancer who were sorafenib-refractory, sorafenib-intolerant, or sorafenib-naïve received one standard dose of pembrolizumab. ${ }^{37}$ Interval results showed an $18 \%$ response rate and a 12.9 month median survival period. ${ }^{37}$ In November of 2018, the Food and Drug Administration granted an accelerated approval for pembrolizumab to be used in treatment of patients with HCC who have been previously treated with sorafenib. ${ }^{37}$ However, in the follow-up, phase III KEYNOTE-240 trial, pembrolizumab failed to meet the primary endpoints for both overall survival and progression-free survival when pembrolizumab was compared to placebo and best supportive care in HCC patients that had already failed systemic therapy. ${ }^{38}$ While pembrolizumab compared with placebo did show improvement in overall survival and progression-free survival, the improvement was not deemed statistically significant (overall survival:hazard ratio, $0.78 ; 95 \%$ confidence interval, $0.611-0.998 ; p=0.0238$; progression-free survival:hazard ratio, $0.78 ; 95 \%$ confidence interval, 0.61-0.99; $p=0.0219) .{ }^{38}$ Although disappointing, this has not deterred other investigations of pembrolizumab in HCC patients. In the UK, a phase II/III study is underway investigating pembrolizumab as an adjunctive treatment to trans-arterial chemoembolization (TACE) using doxorubicin and gelatin sponges. ${ }^{27}$

An additional phase III study, KEYNOTE-394 (NCT03062358), is currently evaluating and recruiting patients and is using the same inclusion criteria as set in the Keynote-240 trial in hopes of better outcomes in an Asian population. ${ }^{39}$

\section{Tislelizumab}

Tislelizumab, another anti-PD-1 antibody, is currently under development by BeiGene. ${ }^{40}$ The safety of tislelizumab was 


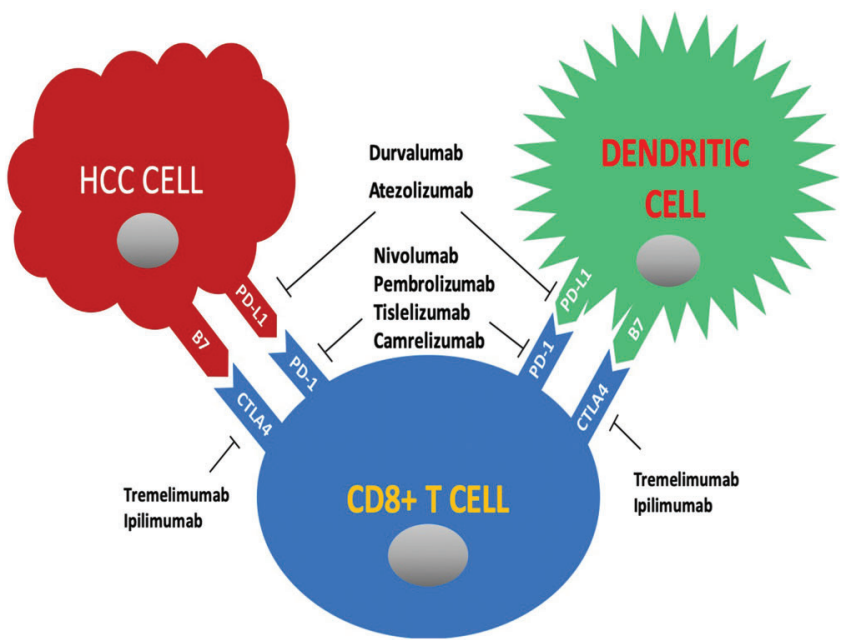

Fig. 2. Mechanism of action of checkpoint inhibitors under investigation for hepatocellular carcinoma treatment.

Hepatocellular carcinoma (HCC) tumor cells can up-regulate expression of programmed cell death-1, PD-1, which binds the programmed death-ligand 1, PD-L1, and stimulates peripheral T cell depletion. ${ }^{29}$ They can also activate cytotoxic $T$ lymphocyte-associated protein-4 (CTLA-4), found on the surface of T cells and leading to down-regulation of $\mathrm{T}$ cells. ${ }^{27}$ Ipilimumab and tremelimumab bind to and inactivate CTLA-4, preventing its activation. PD-1-PD-L1 binding may be prevented by therapeutically blocking either PD-1 (nivolumab, pembrolizumab, tislelizumab, and camrelizumab) or PD-L1 (durvalumab and atezolizumab).

established in an earlier phase I trial, with multiple different solid cancers, including $\mathrm{HCC} .{ }^{41}$ Currently, there is a multicenter global phase III trial looking at tislelizumab versus sorafenib as first-line treatment for unreachable HCC that started recruitment in 2017.27,40,42 This trial set survival rate as the principal endpoint and was designed to validate that non-inferiority of tislelizumab compared to sorafenib (Table 2). No interim data are currently available.

\section{Camrelizumab}

Camrelizumab is an anti-PD-1 antibody, for which a phase II/ III trial is currently underway in China, looking at patients who failed to respond to systemic treatments or were intolerant to previous systemic treatments. ${ }^{27}$ Provisional results from the phase II part was presented in 2018 at a meeting of the European Society for Medical Oncology, demonstrated a response rate of $13.8 \%(30 / 217)$ with a 6 -month overall survival rate of $74.7 \% .{ }^{27}$ It is notable that only two patients $(0.9 \%)$ experienced grade 5 treatment-related adverse events, which in turn showed camrelizumab to have a suitable toxicity profile. ${ }^{27}$ No interim date is available from the phase III part. Currently, there is an ongoing phase II trial in China looking at camrelizumab plus the FOLFOX4 regimen (consisting of 5-fluorouracil, leucovorin, and oxaliplatin) for treatment of advanced HCC and biliary tract cancers in patients who failed systemic treatment. ${ }^{43}$ Interim results from October 2018 were promising, with median progression-free survival of 5.5 months; however, over $85 \%$ of patients had severe treatment related side-effects. ${ }^{43}$

\section{Durvalumab}

Durvalumab is the only anti-PD-L1 antibody for HCC under investigation currently. ${ }^{27}$ Cancer cells can avoid immune surveillance by overexpressing PD-L1 and activating PD-L1/PD-1 signaling, which is observed in HCC tissues (Fig. 2) ${ }^{44,45} \mathrm{~A}$ basic science study recently showed that inhibition of PD-L1 and DNA methyltransferase 1 (commonly known as DNMT1) significantly suppressed the growth of sorafenib-resistant HCC cells in vitro. ${ }^{46,47}$ This points to a possible novel treatment option for sorafenib-resistant HCC. ${ }^{26,48}$ A phase I/II trial looking at the safety of durvalumab monotherapy in treating solid tumors showed durvalumab to have an acceptable safety profile and to be promising, with a $10 \%$ response rate and a median survival time of 13.2 months for the HCC cohort. ${ }^{26}$ A phase III trial that started in 2017 is currently underway looking at durvalumab plus tremelimumab combination therapy as a first-line treatment for patients with advanced HCC; however, currently there are no interim results available. ${ }^{27}$

\section{Tremelimumab}

Tremelimumab is another anti-CTLA-4 antibody under investigation for HCC. ${ }^{27}$ To date, remelimumab monotherapy has been investigated for patients with HCC and chronic hepatitis C. ${ }^{49}$ Results from the trial were promising, and showed that of the 21 patients enrolled, there was a partial response rate of $17.6 \%$ and a median time to progression of 6.48 months. ${ }^{27}$ Overall, the treatment was very well tolerated, with minimal toxicities, such as transient elevation of transaminases, noted. ${ }^{27} \mathrm{~A}$ phase I/II trial investigating the combination of tremelimumab plus interventional procedures, such as radiofrequency ablation, TACE, and cryoablation, for non-resettable HCC patients is currently underway in the USA. ${ }^{30}$ Initial results show no dose limiting toxicities and of the 19 patients that were suitable for evaluation, $5(26.3 \%)$ achieved partial responses outside of the areas treated with TACE or ablation. ${ }^{27}$ The median progression-free survival period was 7.4 months, with the median survival period of 12.3 months. ${ }^{25}$

\section{Combined targeted therapy}

\section{Atezolizumab + bevacizumab}

Novel combination treatment with atezolizumab, an anti-PDL1 antibody, and bevacizumab, an anti-vascular endothelial growth factor (VEGF) therapy, is under investigation for treatment of advanced HCC and has shown to be effective in combination when treating other cancers. ${ }^{50} \mathrm{HCC}$ tumors over-express VEGF and PD-L1 and there is evidence of increased vascularity, which makes it a good target for this combination therapy that targets both sites. ${ }^{51-53}$ Two global I/Ib studies showed promising results when looking at this combo in patients with unrespectable HCC who had not received prior systemic therapy but some of who received TACE and/or radiotherapy. ${ }^{54}$ Data from the non-randomized arm of 119 patients showed an objective response rate of $36 \%$ (95\% confidence interval, 26-46), with $12 \%$ of enrolled patients having complete response to treatment and a median overall survival of 17.1 months. ${ }^{54}$

The second arm of the study randomized the same patient population to atezolizumab and bevacizumab versus atezolizumab monotherapy. ${ }^{54}$ of those who received the 
combination therapy, $20 \%$ had confirmed responses and $47 \%$ had stable disease, compared to $17 \%$ and $32 \%$ of patients, respectively, in the atezolizumab monotherapy group. ${ }^{54}$ This accounted for the 0.55 hazard ratio ( $80 \%$ confidence interval, $0.40-0.74 ; p=0.0108$ ) reported. ${ }^{54}$ Additionally, median progression-free survival in the combination arm was 5.6 months compared to 3.4 months in the monotherapy group. ${ }^{55}$

There were increased grade 3/4 adverse events in the combination group ( $34 \%$ vs. $14 \%$ ) with the most common adverse events being proteinuria, fatigue, and rash. ${ }^{54}$ Based on data that showed an objective response rate of $36 \%$ (95\% confidence interval, 26-46), the Food and Drug Administration granted Breakthrough Therapy Designation for atezolizumab/bevacizumab combination therapy to be a fist-line therapy for advanced or metastatic HCC. ${ }^{55}$ In addition, enrollment for a new phase III study, IMbrave150 (NCT03434379), has completed enrollment and will be comparing atezolizumab/bevacizuma versus sorafenib in unresectable HCC patients. ${ }^{55}$

\section{Ipilimumab + nivolumab}

Ipilimumab is an anti-CTLA-4 antibody and multiple studies have recently investigated its use in combination with nivolumab. In the USA, the CheckMate 040 trial results were recently published (NCT01658878). ${ }^{56}$ The trial enrolled HCC patients who had failed treatment with sorafenib and randomized the patients to receive one of three different protocols of ipilimumab plus nivolumab. The combined treatment reportedly showed an acceptable safety profile, with an objective response rate that was twice that of nivolumab monotherapy ( $31 \%$ compared to $14 \%$ ) and having median overall survival of 18 months. ${ }^{56}$

In the UK, the PRIME-HCC clinical trial is underway, assessing the efficacy of combination treatment pre-operatively with nivolumab and ipilimumab in HCC patients for whom liver resection is planned. ${ }^{27}$ Participants will receive two doses of nivolumab and a single dose of ipilimumab in the weeks before their surgery. This is a single-arm, openlabel study to be conducted in 32 patients at a small number of UK hospitals. The study has two parts. Part 1 will confirm, in a small number of patients, that the treatment regimen is safe and does not result in delay to liver resection, while Part 2 will expand the number of patients studied and assess survival over 2 years post-resection. The decision to proceed to Part 2 will be taken with advice from an independent, expert committee. ${ }^{27}$

\section{SHR-1210 + Apatinib}

The combination of SHR-1210, a novel anti-PD-1 antibody, and apatinib, a tyrosine kinase inhibitor selectively acting on VEGF receptor 2 , is currently being investigated. ${ }^{27}$ A phase 1 trial was completed in 2018 and showed acceptable tolerability of this combination and a response rate of $38.9 \%$, with a median progression-free survival of 7.2 months for the 18 patients with HCC. ${ }^{44}$ Overall, adverse events were relatively tolerable, with only one patient discontinuing the treatment due to treatment-related grade 3 hyperbilirubinemia. ${ }^{44}$ Currently, a phase II trial is underway in the USA, comparing this combination to systemic chemotherapy in advanced HCC. ${ }^{57}$ No results are available currently.

\section{Other targets in immunotherapy}

ACT is a new approach to look for treatments that allow a patients' own lymphocytes to attack cancer cells. ${ }^{58,59}$ Adoptive immunotherapy for HCC includes cytokine-induced killer cells, tumor-infiltrating lymphocytes, natural killer cells, and chimeric antigen receptor (CAR) T cells. The safety of ACT in patients with HCC have been investigated in many preclinical studies, thus laying a foundation for its clinical applications. ${ }^{58,59}$

CAR-T cell therapy, in particular, has been a very successful novel method of treating CD19-positive hematological malignancies, and its application has recently been considered in the treatment of solid tumors, including HCC. ${ }^{33}$ Currently, there are no clinical trials investigating CAR-T cell therapy for HCC, as there have been many concerns about the drug causing cytokine-release syndrome, which affects up to $90 \%$ of patients and can cause cardiovascular, pulmonary, and central nervous system complications. ${ }^{59-61}$ Additionally, there is currently a lack of specific tumor antigens to target in HCC, limited trafficking and penetration of CAR-T cells to tumor sites, and an immunosuppressive tumor microenvironment. ${ }^{33}$ To overcome these difficulties, numerous strategies have been developed, including enhancing the selectivity of CARs and controlling CAR-T activity. ${ }^{33}$ In a recent study by Guo et al., ${ }^{62}$ the investigators concluded that gene-edited CAR T cells with PD-1 deficiency have stronger antitumor activity than wild-type CAR T cells and future development of CAR T cells with modified gene-editing may help improve CAR T cell efficacy as a treatment for HCC. ${ }^{62}$ In another basic research study, Li et al. ${ }^{63}$ demonstrated that GPC3-targeting CAR T cells, in particular CAR.hYP7, are a promising therapeutic intervention for liver cancer that can be translated for human use. ${ }^{63}$ There is hope that with further investigation and clinical trials, CAR-T cell therapy will become a viable option for HCC treatment.

Interferon monotherapy has also been explored as adjuvant therapy, to both prevent tumor recurrence as well as to inhibit development of HCC in patients with chronic hepatitis $B$ and $C$ infections. ${ }^{64-66}$ According to a published report by Lai et al. ${ }^{67}$ interferon- $\alpha$ increased survival rates and encouraged tumor regression in patients diagnosed with advanced HCC. According to Obi et al., ${ }^{68} 16 \%$ of HCC patients with portal vein invasion who received a combination of 5-flurouracil and interferon- $\alpha$ treatment had complete response, while $36 \%$ had a partial response, as interferon- $\alpha$ can induce apoptosis and inhibit cell growth in HCC tumors.

Different than ACT, cell-mediated immunotherapy is a novel approach that has been used to exploit the unique pattern of proteins that are expressed specifically on tumor cells as targets. Tumor-targeted antibodies are mutant or aberrantly expressed antigens on the surface on cancer cells and can be potential targets of the adaptive human immune system. ${ }^{36}$ Tumor-targeted antibodies are being investigated for HCC immunotherapy, with a focus on alpha feto-proteindirected treatments. ${ }^{69}$ Another approach is to use oncolytic viruses to attack HCC tumor cells. ${ }^{70-72}$ Theoretically, these viruses can selectively replicate in tumor cells and cause lysis without harming normal tissues. ${ }^{26}$ Oncolytic virotherapy-mediated oncolysis not only leads to tumor regression but also provides important immune responses. Most investigations into oncolytic virotherapy are currently in the preclinical or early clinical stages but are promising. ${ }^{70}$ 


\section{Conclusions}

Immunotherapy is a promising new frontier for HCC treatment, with many novel new strategies currently under development. The incidence of HCC is rapidly increasing, and it is a major cause of morbidity and mortality both nationally and internationally. Though HCC can be a devastating disease, the best hope for prolonged survival is early screening and diagnosis. Although there is some debate regarding the ideal methods of surveillance, ultrasound with and without alpha feto-protein every 6-12 months is generally accepted as standard practice in the proper patient population. Currently, multiple therapeutic modalities are available and research investigating innovative options is ongoing. Most patients are best served in liver transplant centers, where a multidisciplinary approach can take place under the guidance of experienced transplant hepatologists and gastroenterologists. Advances in HCC prevention, detection, and treatments have resulted in improved survival for a disease that was, until recently, considered terminal. Randomized phase I-III trials of nivolumab, atezolizumab, durvalumab, ipilimumab and tislelizumab as monotherapy or combination therapy are currently being conducted. However, there is still much to be revealed regarding checkpoint inhibitors as well as immunotherapy involving gut microbiota and monocytes in the peripheral blood, so clinical trials are necessary to determine their full benefit. Immunotherapy and targeted molecular therapies have personalized medical therapy, while improving patient care, and hopefully future research can continue this endeavor.

\section{Funding}

None to declare.

\section{Conflict of interest}

Nikolaos T. Pyrsopoulos declares separate research grants from Allergan, Bayer, Beigine, Bristol Myers Squibb, Confirm, Conatus, Intercept, Mallinckrodt, Novartis, Resusix, Saro, Valeant, Gilead, Exelixis, Hologic, Shire, Genfit and Prometheus. Also, Nikolaos T. Pyrsopoulos is an advisory consultant for Bayer, Exelixis, Gilead and Novartis. The other authors have no conflict of interests related to this publication.

\section{Author contributions}

All authors contributed equally to this paper in conception and design of the study, literature review and analysis, drafting, critical revision, editing, and giving approval of the final version.

\section{References}

[1] Bray F, Ferlay J, Soerjomataram I, Siegel RL, Torre LA, Jemal A. Global cancer statistics 2018: GLOBOCAN estimates of incidence and mortality worldwide for 36 cancers in 185 countries. CA Cancer J Clin 2018;68:394-424. doi: 10. 3322/caac. 21492.

[2] Waller LP, Deshpande V, Pyrsopoulos N. Hepatocellular carcinoma: A comprehensive review. World J Hepatol 2015;7:2648-2663. doi: 10.4254/wjh.v7. i26.2648.

[3] Lai SW. Risk factors for hepatocellular carcinoma. Cancer 2019;125:482. doi: $10.1002 /$ cncr 31802
[4] Patel P, Schutzer SE, Pyrsopoulos N. Immunobiology of hepatocarcinogenesis: Ways to go or almost there? World J Gastrointest Pathophysiol 2016;7:242255. doi: 10.4291/wjgp.v7.i3.242.

[5] Sucandy I, Cheek S, Tsung A, Marsh JW, Geller DA. Minimally invasive liver resection for primary and metastatic liver tumors: influence of age on perioperative complications and mortality. Surg Endosc 2018;32:1885-1891. doi: 10.1007/s00464-017-5880-7.

[6] Wang J, Xu L, Zeng W, Hu P, Zeng M, Rabkin SD, Liu R. Treatment of human hepatocellular carcinoma by the oncolytic herpes simplex virus G47delta. Cancer Cell Int 2014;14:83. doi: 10.1186/s12935-014-0083-y.

[7] Wang G, Zhu S, Li X. Comparison of values of CT and MRI imaging in the diagnosis of hepatocellular carcinoma and analysis of prognostic factors. Oncol Lett 2019;17:1184-1188. doi: 10.3892/ol.2018.9690.

[8] Ayuso C, Rimola J, Vilana R, Burrel M, Darnell A, García-Criado Á, et al. Corrigendum to "Diagnosis and staging of hepatocellular carcinoma (HCC): Current guidelines" [Eur. J. Radiol. 101 (2018) 72-81]. Eur J Radiol 2019; 112:229. doi: 10.1016/j.ejrad.2019.01.018.

[9] Lee MW, Lim HK. Management of sub-centimeter recurrent hepatocellular carcinoma after curative treatment: Current status and future. World J Gastroenterol 2018;24:5215-5222. doi: 10.3748/wjg.v24.i46.5215.

[10] Corrigendum to "EASL Clinical Practice Guidelines: Management of hepatocellular carcinoma" [J Hepatol 69 (2018) 182-236]. J Hepatol 2019;70:817. doi: $10.1016 /$ j.jhep.2019.01.020.

[11] EASL Clinical Practice Guidelines: Management of hepatocellular carcinoma J Hepatol 2018;69:182-236. doi: 10.1016/j.jhep.2018.03.019.

[12] Keane FK, Hong TS, Zhu AX. Evolving systemic therapy in hepatocellular carcinoma: current management and opportunities for integration with radiotherapy. Semin Radiat Oncol 2018;28:332-341. doi: 10.1016/j.semradonc.2018.06.006.

[13] Holzwanger DJ, Madoff DC. Role of interventional radiology in the management of hepatocellular carcinoma: current status. Chin Clin Oncol 2018;7 49. doi: 10.21037/cco.2018.07.04

[14] Brar G, Greten TF, Brown ZJ. Current frontline approaches in the management of hepatocellular carcinoma: the evolving role of immunotherapy. Therap Adv Gastroenterol 2018;11:1756284818808086. doi: 10 $1177 / 1756284818808086$

[15] Chhatwal J, Wang X, Ayer T, Kabiri M, Chung RT, Hur C, et al. Hepatitis C disease burden in the United States in the era of oral direct-acting antivirals. Hepatology 2016;64:1442-1450. doi: 10.1002/hep.28571

[16] Hasegawa K, Kokudo N, Makuuchi M, Izumi N, Ichida T, Kudo M, et al. Comparison of resection and ablation for hepatocellular carcinoma: a cohor study based on a Japanese nationwide survey. J Hepatol 2013;58:724729. doi: 10.1016/j.jhep.2012.11.009.

[17] Rich NE, Parikh ND, Singal AG. Hepatocellular carcinoma and liver transplantation: Changing patterns and practices. Curr Treat Options Gastroentero 2017; 15:296-304. doi: 10.1007/s11938-017-0133-3.

[18] Walker ND, Mourad Y, Liu K, Buxhoeveden M, Schoenberg C, Eloy JD, et al. Steroid-mediated decrease in blood mesenchymal stem cells in liver transplant could impact long-term recovery. Stem Cell Rev Rep 2017;13:644658. doi: 10.1007/s12015-017-9751-3.

[19] Nitta H, Allard MA, Sebagh M, Karam V, Ciacio O, Pittau G, et al. Predictive model for microvascular invasion of hepatocellular carcinoma among candidates for either hepatic resection or liver transplantation. Surgery $2019 ; 165$ 1168-1175. doi: 10.1016/j.surg.2019.01.012.

[20] Liver transplantation and hepatic resection can achieve cure for hepatocellular carcinoma: Erratum. Ann Surg 2019;269:e59. doi: 10.1097/SLA. 0000000000003141

[21] Kulik L, El-Serag HB. Epidemiology and management of hepatocellular carcinoma. Gastroenterology 2019;156:477-491.e1. doi: 10.1053/j.gastro. 2018.08.065.

[22] Samuel D, Coilly A. Management of patients with liver diseases on the waiting list for transplantation: a major impact to the success of liver transplantation. BMC Med 2018;16:113. doi: 10.1186/s12916-018-1110-y.

[23] Okoronkwo N, Wang Y, Pitchumoni C, Koneru B, Pyrsopoulos N. Improved outcomes following hepatocellular carcinoma (HCC) diagnosis in patient screened for $\mathrm{HCC}$ in a large academic liver center versus patients identified in the community. J Clin Transl Hepatol 2017;5:31-34. doi: 10.14218/JCTH. 2016.00051.

[24] Olivo R, Guarrera JV, Pyrsopoulos NT. Liver Transplantation for Acute Liver Failure. Clin Liver Dis 2018;22:409-417. doi: 10.1016/j.cld.2018.01.014.

[25] Pinter M, Peck-Radosavljevic M. Review article: systemic treatment of hepatocellular carcinoma. Aliment Pharmacol Ther 2018;48:598-609. doi: 10 1111/apt.14913.

[26] Xie Y, Xiang Y, Sheng J, Zhang D, Yao X, Yang Y, Zhang X. Immunotherapy for hepatocellular carcinoma: Current advances and future expectations. J Immunol Res 2018:2018:8740976. doi: 10.1155/2018/8740976.

[27] Okusaka T, Ikeda M. Immunotherapy for hepatocellular carcinoma: current status and future perspectives. ESMO Open 2018;3:e000455. doi: 10. 1136/esmoopen-2018-000455. 
[28] Schreiber RD, Old L], Smyth MJ. Cancer immunoediting: integrating immunity's roles in cancer suppression and promotion. Science 2011;331:15651570. doi: 10.1126/science.1203486.

[29] Tumeh PC, Harview CL, Yearley JH, Shintaku IP, Taylor EJ, Robert L, et al. PD1 blockade induces responses by inhibiting adaptive immune resistance. Nature 2014;515:568-571. doi: 10.1038/nature13954.

[30] Duffy AG, Ulahannan SV, Makorova-Rusher O, Rahma O, Wedemeyer H, Pratt D, et al. Tremelimumab in combination with ablation in patients with advanced hepatocellular carcinoma. J Hepatol 2017;66:545-551. doi: 10.1016/j.jhep. 2016.10.029.

[31] Iñarrairaegui M, Melero I, Sangro B. Immunotherapy of hepatocellular carcinoma: facts and hopes. Clin Cancer Res 2018;24:1518-1524. doi: 10. 1158/1078-0432.CCR-17-0289.

[32] El-Khoueiry AB, Sangro B, Yau T, Crocenzi TS, Kudo M, Hsu C, et al. Nivolumab in patients with advanced hepatocellular carcinoma (CheckMate 040): an open-label, non-comparative, phase $1 / 2$ dose escalation and expansion trial. Lancet 2017;389:2492-2502. doi: 10.1016/S0140-6736(17)31046-2.

[33] Chen Y, E CY, Gong ZW, Liu S, Wang ZX, Yang YS, Zhang XW. Chimeric antigen receptor-engineered T-cell therapy for liver cancer. Hepatobiliary Pancreat Dis Int 2018;17:301-309. doi: 10.1016/j.hbpd.2018.05.005.

[34] Bristol-Myers Squibb announces results from CheckMate -459 Study Evaluating Opdivo (nivolumab) as a first-line treatment for patients with unresectable hepatocellular carcinoma. Available from: https://news.bms. com/press-release/bmy/bristol-myers-squibb-announces-results-checkmate-459-study-evaluating-opdivo-nivol.

[35] Contratto M, Wu J. Targeted therapy or immunotherapy? Optimal treatment in hepatocellular carcinoma. World J Gastrointest Oncol 2018;10:108-114. doi: $10.4251 /$ wjgo.v10.i5.108.

[36] Eso Y, Marusawa H. Novel approaches for molecular targeted therapy against hepatocellular carcinoma. Hepatol Res 2018;48:597-607. doi: 10. 1111/hepr.13181.

[37] Zhu AX, Finn RS, Edeline J, Cattan S, Ogasawara S, Palmer D, et al. Pembrolizumab in patients with advanced hepatocellular carcinoma previously treated with sorafenib (KEYNOTE-224): a non-randomised, open-label phase 2 trial. Lancet Oncol 2018;19:940-952. doi: 10.1016/S1470-2045 (18)30351-6.

[38] Merck provides update on KEYNOTE-240, a phase 3 study of KEYTRUDA ${ }^{\circledR}$ (pembrolizumab) in previously treated patients with advanced hepatocellular carcinoma. Available from: https://investors.merck.com/news/pressrelease-details/2019/Merck-Provides-Update-on-KEYNOTE-240-a-Phase-3Study-of-KEYTRUDA-pembrolizumab-in-Previously-Treated-Patients-withAdvanced-Hepatocellular-Carcinoma/default.aspx.

[39] Xu W, Liu K, Chen M, Sun JY, McCaughan GW, Lu XJ, et al. Immunotherapy for hepatocellular carcinoma: recent advances and future perspectives. Ther Adv Med Oncol 2019;11:1758835919862692. doi: 10.1177/1758835919862692.

[40] Qin S, Finn RS, Kudo M, Meyer T, Vogel A, Ducreux M, et al. A phase 3 , randomized, open-label, multicenter study to compare the efficacy and safety of tislelizumab, an anti-PD-1 antibody, versus sorafenib as first-line treatment in patients with advanced hepatocellular carcinoma. J Clin Oncol 2018;36:TPS3110-TPS3110. doi: 10.1200/JCO.2018.36.15_suppl. TPS3110.

[41] Finkelmeier F, Waidmann O, Trojan J. Nivolumab for the treatment of hepatocellular carcinoma. Expert Rev Anticancer Ther 2018;18:1169-1175. doi: 10.1080/14737140.2018.1535315.

[42] Ikeda M, Sung MW, Kudo M, Kobayashi M, Baron AD, Finn RS, et al. A phase $1 \mathrm{~b}$ trial of lenvatinib (LEN) plus pembrolizumab (PEM) in patients (pts) with unresectable hepatocellular carcinoma (uHCC). J Clin Oncol 2018;36:4076. doi: 10.1200/JCO.2018.36.15_suppl.4076.

[43] Qin S, Chen Z, Liu Y, Xiong J, Ren Z, Meng Z, et al. A phase II study of antiPD-1 antibody camrelizumab plus FOLFOX4 or GEMOX systemic chemotherapy as first-line therapy for advanced hepatocellular carcinoma or biliary tract cancer. J Clin Oncol 2019;37:4074. doi: 10.1200/JCO.2019.37. 15_suppl.4074.

[44] Iwai $Y$, Ishida M, Tanaka $Y$, Okazaki T, Honjo T, Minato N. Involvement of PD-L1 on tumor cells in the escape from host immune system and tumor immunotherapy by PD-L1 blockade. Proc Natl Acad Sci U S A 2002;99: 12293-12297. doi: 10.1073/pnas.192461099.

[45] Zhong F, Cheng X, Sun S, Zhou J. Transcriptional activation of PD-L1 by Sox2 contributes to the proliferation of hepatocellular carcinoma cells. Oncol Rep 2017;37:3061-3067. doi: 10.3892/or.2017.5523.

[46] Colombo M, Lleo A. Is Liver Injury an Affordable Risk of Immune Checkpoint Inhibitor Therapy for Cancer? Gastroenterology 2018;155:2021-2023. doi: 10.1053/j.gastro.2018.11.016.

[47] Xu F, Jin T, Zhu Y, Dai C. Immune checkpoint therapy in liver cancer. J Exp Clin Cancer Res 2018;37:110. doi: 10.1186/s13046-018-0777-4.

[48] Liu J, Liu Y, Meng L, Liu K, Ji B. Targeting the PD-L1/DNMT1 axis in acquired resistance to sorafenib in human hepatocellular carcinoma. Oncol Rep 2017; 38:899-907. doi: 10.3892/or.2017.5722.

[49] Sangro B, Gomez-Martin C, de la Mata M, Iñarrairaegui M, Garralda $E_{\text {, }}$ Barrera $\mathrm{P}$, et al. A clinical trial of CTLA-4 blockade with tremelimumab in patients with hepatocellular carcinoma and chronic hepatitis C. J Hepatol 2013;59:81-88. doi: 10.1016/j.jhep.2013.02.022.

[50] Zhu AX. New agents on the horizon in hepatocellular carcinoma. Ther Adv Med Oncol 2013;5:41-50. doi: 10.1177/1758834012458480.

[51] Stein S, Pishvaian MJ, Lee MS, Lee KH, Hernandez S, Kwan A, et al. Safety and clinical activity of $1 \mathrm{~L}$ atezolizumab + bevacizumab in a phase Ib study in hepatocellular carcinoma (HCC). J Clin Oncol 2018;36:4074. doi: 10. 1200/JCO.2018.36.15_suppl.4074.

[52] Finn RS, Ducreux M, Qin S, Galle PR, Zhu AX, Ikeda M, et al. IMbrave150: A randomized phase III study of $1 \mathrm{~L}$ atezolizumab plus bevacizumab vs sorafenib in locally advanced or metastatic hepatocellular carcinoma. J Clin Oncol 2018;36:TPS4141. doi: 10.1200/JCO.2018.36.15_suppl.TPS4141.

[53] Pishvaian MJ, Lee MS, Ryoo BY, Stein S, Lee KH, Liu B, et al. LBA26 Updated safety and clinical activity results from a phase Ib study of atezolizumab + bevacizumab in hepatocellular carcinoma (HCC). Ann Oncol 2018;29: viii718-viii719. doi: 10.1093/annonc/mdy424.028.

[54] Lee M, Ryoo BY, Hsu CH, Numata K, Stein S, Verret W, et al. LBA39- Randomised efficacy and safety results for atezolizumab (Atezo) + bevacizumab (Bev) in patients (pts) with previously untreated, unresectable hepatocellular carcinoma (HCC). Ann Oncol 2019;30:v875. doi: 10. 1093/annonc/mdz394.030.

[55] Roche's Tecentriq in combination with Avastin shows encouraging results in Phase Ib study of people with unresectable hepatocellular carcinoma. Available from: https://www.roche.com/investors/updates/inv-update-2019-0927.htm.

[56] Yau T, Kang YK, Kim TY, El-Khoueiry AB, Santoro A, Sangro B, et al. Nivolumab (NIVO) + ipilimumab (IPI) combination therapy in patients (pts) with advanced hepatocellular carcinoma (aHCC): Results from CheckMate 040. J Clin Oncol 2019;37:4012. doi: 10.1200/JCO.2019.37.15 suppl.4012.

[57] Xu J, Zhang Y, Jia R, Yue C, Chang L, Liu R, et al. Anti-PD-1 antibody SHR1210 combined with apatinib for advanced hepatocellular carcinoma, gastric, or esophagogastric junction cancer: An open-label, dose escalation and expansion study. Clin Cancer Res 2019;25:515-523. doi: 10.1158/10780432.CCR-18-2484.

[58] Jafferji MS, Yang JC. Adoptive T-cell therapy for solid malignancies. Surg Oncol Clin N Am 2019;28:465-479. doi: 10.1016/j.soc.2019.02.012.

[59] Met Ö, Jensen KM, Chamberlain CA, Donia M, Svane IM. Principles of adoptive T cell therapy in cancer. Semin Immunopathol 2019;41:49-58. doi: 10. 1007/s00281-018-0703-z.

[60] Neelapu SS. Managing the toxicities of CAR T-cell therapy. Hematol Oncol 2019;37 Suppl 1:48-52. doi: 10.1002/hon.2595.

[61] Yakoub-Agha I, Moreau AS, Ahmad I, Borel C, Hadhoum N, Masouridi-Levrat $\mathrm{S}$, et al. Prise en charge pratique du syndrome de relargage des cytokines (CRS) post-CAR-T cells chez l'adulte et l'enfant : recommandation de la Société francophone de greffe de moelle et de thérapie cellulaire (SFGMTC) [Management of cytokine release syndrome in adult and pediatric patients undergoing CAR-T cell therapy for hematological malignancies: Recommendation of the French Society of Bone Marrow and cellular Therapy (SFGM-TC)]. Bull Cancer 2019;106:S102-S109. French. doi: 10.1016/j. bulcan.2018.12.001.

[62] Guo X, Jiang H, Shi B, Zhou M, Zhang H, Shi Z, et al. Disruption of PD-1 enhanced the anti-tumor activity of chimeric antigen receptor $\mathrm{T}$ cells against hepatocellular carcinoma. Front Pharmacol 2018;9:1118. doi: 10. 3389/fphar.2018.01118.

[63] Li D, Li N, Zhang Y, Fu H, Torres MB, Wang Q, et al. Development of CAR T-cell therapy targeting glypican-3 in liver cancer [abstract]. In: Proceedings of the American Association for Cancer Research Annual Meeting 2018; 2018 Apr 14-18; Chicago, IL. Philadelphia (PA): AACR; Cancer Res 2018;78(13 Suppl): Abstract nr 2549. doi: 10.1158/1538-7445.AM2018-2549.

[64] Nishibatake Kinoshita M, Minami T, Tateishi R, Wake T, Nakagomi R, Fujiwara $\mathrm{N}$, et al. Impact of direct-acting antivirals on early recurrence of HCV-related HCC: Comparison with interferon-based therapy. J Hepatol 2019;70:78-86. doi: 10.1016/j.jhep.2018.09.029.

[65] Toyoda H, Kumada T, Tada T, Mizuno K, Sone Y, Kaneoka Y, et al. Impact of previously cured hepatocellular carcinoma (HCC) on new development of HCC after eradication of hepatitis C infection with non-interferon-based treatments. Aliment Pharmacol Ther 2018;48:664-670. doi: 10.1111/apt. 14914.

[66] Tsai PC, Huang CF, Yu ML. Unexpected early tumor recurrence in patients with hepatitis $C$ virus-related hepatocellular carcinoma undergoing interferon-free therapy: Issue of the interval between HCC treatment and antiviral therapy. J Hepatol 2017;66:464. doi: 10.1016/j.jhep.2016.10.035.

[67] Lai CL, Lau JY, Wu PC, Ngan H, Chung HT, Mitchell SJ, et al. Recombinant interferon-alpha in inoperable hepatocellular carcinoma: a randomized controlled trial. Hepatology 1993;17:389-394. doi: 10.1002/hep.1840170307.

[68] Obi S, Yoshida H, Toune R, Unuma T, Kanda M, Sato $S$, et al. Combination therapy of intraarterial 5-fluorouracil and systemic interferon-alpha for advanced hepatocellular carcinoma with portal venous invasion. Cancer 2006;106:1990-1997. doi: 10.1002/cncr.21832. 
[69] Butterfield LH, Ribas A, Meng WS, Dissette VB, Amarnani S, Vu HT, et al. Tcell responses to HLA-A*0201 immunodominant peptides derived from alpha-fetoprotein in patients with hepatocellular cancer. Clin Cancer Res 2003;9:5902-5908.

[70] Yoo SY, Badrinath N, Woo HY, Heo J. Oncolytic virus-based immunotherapies for hepatocellular carcinoma. Mediators Inflamm 2017;2017:5198798. doi: $10.1155 / 2017 / 5198798$.

[71] Breitbach CJ, Moon A, Burke J, Hwang TH, Kirn DH. A phase 2, open-label, randomized study of pexa-vec (JX-594) administered by intratumoral injection in patients with unresectable primary hepatocellular carcinoma. Methods Mol Biol 2015;1317:343-357. doi: 10.1007/978-1-49392727-2_19.
[72] Ady JW, Heffner J, Mojica $K$, Johnsen $C$, Belin LJ, Love $D$, et al. Oncolytic immunotherapy using recombinant vaccinia virus GLV-1h68 kills sorafenibresistant hepatocellular carcinoma efficiently. Surgery 2014;156:263-269. doi: 10.1016/j.surg.2014.03.031.

[73] Wainberg ZA, Segal NH, Jaeger D, Lee KH, Marshall J, Antonia SJ, et al. Safety and clinical activity of durvalumab monotherapy in patients with hepatocellular carcinoma (HCC). J Clin Oncol 2017;35:4071. doi: 10.1200/JCO. 2017.35.15 suppl.4071.

[74] Kelley RK, Abou-Alfa GK, Bendell JC, Kim TY, Borad MJ, Yong WP, et al. Phase I/II study of durvalumab and tremelimumab in patients with unresectable hepatocellular carcinoma (HCC): Phase I safety and efficacy analyses. J Clin Oncol 2017;35:4073. doi: 10.1200/JCO.2017.35.15_suppl.4073. 